\title{
Synthesis and antibacterial activity of new lactone 1,4- dihydroquinoline derivatives
}

\author{
Rosangela S. Laurentiz $\mathbb{D}^{1}$ - Willian P. Gomes ${ }^{1} \cdot$ Ana P. R. Pissurno $^{1} \cdot$ Fernanda A. Santos $^{1}$. \\ Vinicius Cristian O. Santos ${ }^{2} \cdot$ Carlos H. G. Martins $^{2}$
}

Received: 31 August 2017 / Accepted: 22 December 2017 / Published online: 2 February 2018

(c) Springer Science+Business Media, LLC, part of Springer Nature 2018

\begin{abstract}
In this work, a series of lactone 1,4-dihydroquinoline derivatives $\mathbf{4}$ were efficiently synthesized and characterized by ${ }^{1} \mathrm{H}$ and ${ }^{13} \mathrm{C}$ NMR. The synthesized compounds were evaluated for their in vitro antibacterial activity against the bacterial strains Porphyromonas gingivalis, Prevotella nigrescens, Streptococcus mitis, and Streptococcus sanguinis and against Mycobacterium tuberculosis, Mycobacterium avium and Mycobacterium kansasii. The results revealed that the evaluated compounds were more active against Gram negative bacteria. Compounds $4 \mathbf{b a}, 4 \mathbf{b b}, 4 \mathbf{b g}, \mathbf{4 b i}, \mathbf{4 b n}, \mathbf{4 c h}$, and $4 \mathbf{c i}$ displayed moderate antibacterial activity against $P$. gingivalis. 4bi was the most active compound against the three strains of Mycobacterium. Based on structure-activity relationship studies, we observed that the presence of a nitro group on the benzylic ring and a methylenedioxy group on the dihydroquinoline ring enhanced the antibacterial activity of the derivatives.
\end{abstract}

Keywords Azo-heterocyclic compounds $\cdot$ quinoline derivatives $\cdot$ antibacterial activity

\section{Introduction}

The combination of pharmacophoric moieties of different bioactive substances can produce a new hybrid molecule with improved biological efficacy and affinity, a modified selectivity profile, and different and/or dual modes of action, and may reduce undesired side effects (ViegasJunior et al. 2007). The combination of lactone and 1,4dihydroquinoline rings provides 7-azo synthetic analogs of aryltetralin lignan lactones. Aryltetralin lignan lactones are among the various classes of naturally occurring bioactive molecules that have aroused interest in the area of medicinal chemistry due to their structural diversity and biological

Electronic supplementary material The online version of this article (https://doi.org/10.1007/s00044-017-2129-x) contains supplementary material, which is available to authorized users.

Rosangela S. Laurentiz

rosangela@dfq.feis.unesp.br

1 Department of Physical and Chemistry, Universidade Estadual Paulista Júlio de Mesquista Filho (UNESP), Ilha Solteria, São Paulo, Brazil

2 Research Laboratory in Applied Microbiology, Universidade de Franca, Franca, São Paulo, Brazil properties (Teponno et al. 2016). Several studies have shown that the biological properties of these lignans are closely related to the nature of ring A, their stereochemistry, and substituents on the aromatic rings (Antunez-Mojica et al. 2016). However, little is known about how modifications in the chemical skeleton of ring B can affect these properties. The replacement of $\mathrm{C} 7$ with nitrogen can greatly alter these properties, creating new analogs with biological properties that have not yet been described for this class of compounds (Fig. 1). These derivatives present two important pharmacophoric groups, the lactone and dihydroquinoline rings. The lactone ring is present in many compounds with biological properties (Qiu et al. 2016) including antimicrobials (Grabarczyk et al. 2013; Mazur et al. 2016), along with quinoline and dihydroquinoline rings (Meléndez-Gómez and Kouznetsov 2013; Kharb and Kaur 2013; Desai et al. 2017).

New quinoline derivatives have been synthesized and investigated for several biological properties. The position and type of the substituents on the quinoline and dihydroquinoline rings are responsible for the variety of pharmacological activities that these compounds present, including antibacterial (El-Essawy and El-Sayed 2013; Desai et al. 2017), antiplasmodial (Vandekerckhove et al. 2015), antituberculosis (Keri and Patil 2014), antimalarial (Vandekerckhove and D'hooghe 2015) and anticancer 
Fig. 1 General structure of dihydroquinoline ring, aryltetralin lignan lactone and lactone quinoline derivative

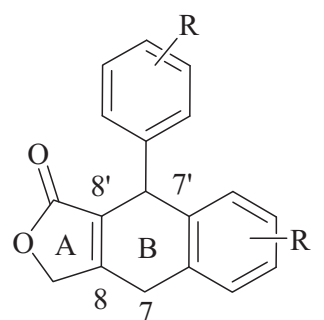

Aryltetralin lignan lactone<smiles>[R]C1=C([R])C([R])c2cc([R])ccc2N1</smiles>

Dihydroquinoline ring<smiles>[R][X]c1ccccc1C1C2=C(COC2=O)Nc2ccccc21</smiles>

Lactone quinoline derivative
Scheme 1 Reaction conditions for obtaining derivatives 4

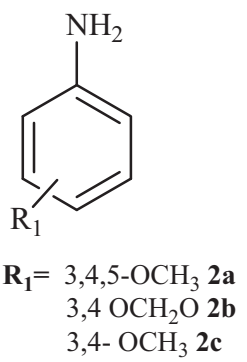<smiles>[R2]c1ccc(C=O)cc1</smiles>

$\mathbf{R}_{\mathbf{2}}=3,4-\left(\mathrm{OCH}_{2} \mathrm{O}\right) \mathbf{3 a}, 3,4-\left(\mathrm{OCH}_{3}\right) \mathbf{3 b}$

$3,4,5-\left(\mathrm{OCH}_{3}\right)$ 3c, $4-\mathrm{Cl} \mathbf{3 d}$

3-( $\left(\mathrm{OCH}_{3}\right)-4 \mathrm{OBn} 3 \mathbf{e}, 4-\mathrm{F} 3 \mathbf{f}$

$4-\mathrm{SCH}_{3} 3 \mathbf{3 g} ; 4-\mathrm{CF}_{3} \mathbf{3 h}$

$3,4-\left(\mathrm{OCH}_{3}\right)_{2}-6-\mathrm{NO}_{2} \mathbf{3 i}$

$3-\left(\mathrm{OCH}_{3}\right)-4-(\mathrm{OH}) \mathbf{3 j}, 3-(\mathrm{OH}) \mathbf{3 k}$

$3-\left(\mathrm{OCH}_{3}\right) 2 \mathrm{l}, 3,4-(\mathrm{OH}) 3 \mathbf{m}$

$3,4-\mathrm{OCH}_{2} \mathrm{O}-6-\mathrm{NO}_{2}$ 3n
(Spanò et al. 2015). However, there are no reports in the literature on the antimicrobial activity of lactone dihydroquinoline derivatives; therefore we decided to synthesize these compounds and evaluate their antibacterial activities.

\section{Result and Discussion}

\section{Chemistry}

The lactone dihydroquinoline derivatives $\mathbf{4}$ presented in this paper were prepared via a microwave-assisted reaction between tetronic acid $\mathbf{1}$, aniline $\mathbf{2}$ and aromatic aldehyde $\mathbf{3}$ in $\mathrm{EtOH}$ (Scheme 1). The use of a microwave furnished the derivatives 4 in high yield and with a shorter reaction time than the traditional methodology (Frackenpohl et al. 2009). The reaction was realized with anilines $\mathbf{2 a - c}$ and aromatic aldehydes 3b-n with electron-withdrawing, as well as electron-donating substituents in different positions on the aromatic ring (Scheme 1).

Substitution of aldehydes with a nitro group (ortho position) furnished the derivatives $\mathbf{4}$ in lower yields, due to the steric hindrance that the nitro group causes on the carbonyl. More pronounced effects on the reaction were observed depending on the substituent present in the aniline. Anilines with electron-withdrawing substituents or without electron-donating substituents in the meta position did not react in these conditions. According to the reactional mechanism proposed herein (Scheme 2), the reaction might proceed via sequential condensation, addition, cyclization, and elimination. First, condensation takes place between the tetronic acid $\mathbf{1}$ with aromatic aldehydes $\mathbf{3}$ to afford the intermediate I. The Michael addition of aniline $\mathbf{2}$ in $\mathbf{I}$ then furnishes the intermediate product II, which isomerizes to III.

In the intermediate III, the nucleophilic addition of the nitrogen in the carbonyl leads to the formation of the intermediate IV, which then undergoes dehydration to generate the target derivative 4 . In this proposed mechanism, the donating group in the meta position in the aniline activates the aromatic ring (ortho position at $\mathrm{NH}_{2}$ group) to attack at intermediate I. The proposed mechanism explains the low reactivity of anilines with electron-withdrawing, as well as weakly electron-donating substituents in the para or ortho positions.

The combination of several anilines and aldehydes furnished 39 lactone dihydroquinoline derivatives, whose structures were confirmed by ${ }^{1} \mathrm{H}$ and ${ }^{13} \mathrm{C}$ NMR spectra. The 
Scheme 2 Mechanistic proposal for the formation of 4

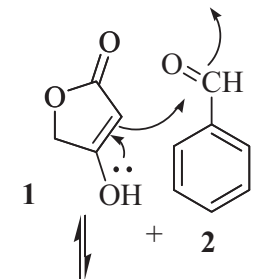

$\underbrace{11}_{0}$<smiles>[R][R]c1ccc([Y])c(C2C3=C(COC3=O)Nc3ccccc32)c1</smiles>

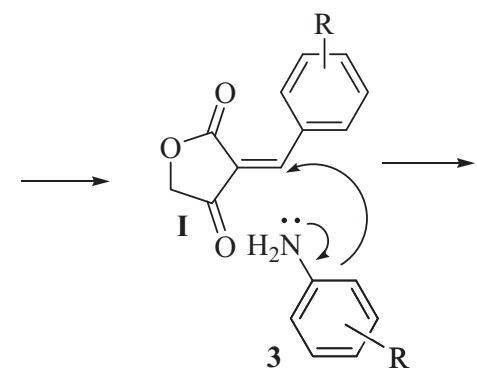<smiles>[R]Cc1cccc(C(C2C=CC=CC2=N)C2C(=O)COC2=O)c1</smiles>

II<smiles>C#CI</smiles>

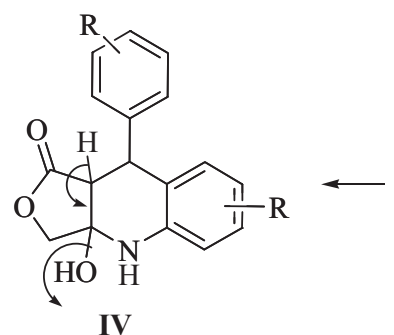

spectral analyses were consistent with the structures proposed and are listed in the supplementary material.

\section{Antibacterial activity}

The 39 compounds synthesized were evaluated in vitro for antibacterial activity against Gram negative bacteria, Gram positive bacteria and mycobacterium strains by the microplate microdilution method using resazurin as an indicator of microbial activity. The minimum inhibitory concentration (MIC) values of antibacterial activity are shown in Table 1. The evaluated compounds were most active against Gram negative bacteria. $P$. gingivalis was more sensitive to the compounds evaluated than $P$. nigrescence. The substituents of aniline and aldehyde, such as halogen, methoxy, trifluormethyl, hydroxy, nitro, and methylthio were varied to explore the structure-activity relationships between the lactone dihydroquinoline derivatives. The results of the evaluation indicated that in the dihydroquinoline ring methylenedioxy and dimethoxy substituents were more favorable for antibacterial activity than trimethoxy substituents. In the benzylic ring, methylenedioxy, dimethoxy, methylthio, trifluormethyl, and nitro substituents favored antibacterial activity. Compounds $4 \mathrm{ba}, 4 \mathbf{b b}, 4 \mathrm{bg}, 4 \mathrm{bi}, 4 \mathbf{b n}$, $\mathbf{4 c h}$, and $\mathbf{4 c i}$ showed good antibacterial activity against $P$. gingivalis. Only compounds $\mathbf{4 a b}, \mathbf{4} \mathbf{a e}$ were active against $P$. nigrescence. None of the compounds evaluated were active against Gram positive bacteria. Compound 4bi was the most active against the three strains of Mycobacterium. Overall, the most active compounds were $4 \mathbf{b i}$ and $4 \mathbf{b n}$, which possess nitro and electron-donating groups attached to the benzylic ring and a methylenedioxy group attached to the dihydroquinoline ring. Nitro-organic compounds are reduced by microbial systems and reduced derivatives were shown to be reactive and to bind to DNA and to proteins (Shahid et al. 2016). However, the antibacterial effect of compounds $4 \mathbf{b i}$ and $\mathbf{4 b n}$ depend not only on the nitro group, but also on their overall structure and the type of bacteria evaluated.

\section{Conclusion}

Thirty-nine lactone dihydroquinoline derivatives were synthesized and their antibacterial activities against anaerobic bacteria, aerobic bacteria, and mycobacterium were assayed using the microplate microdilution method. The presence of halogen or hydroxyl groups did not enhance their antibacterial activity. The most active compounds were $4 \mathbf{b i}$ and 4bn, which possess nitro and electron-donating groups attached to the benzylic ring and a methylenedioxy group attached to the dihydroquinoline ring. Therefore, for this class of compounds the presence of these substituents was essential for antibacterial activity against $P$. gingivalis and Mycobacterium.

\section{Materials and Methods}

\section{Chemistry}

All chemicals and solvents were purchased from commercial sources and were used as received without further purification. Microwave irradiation was carried out with a 
Table 1 In vitro antibacterial activity (MIC $\mu \mathrm{g} / \mathrm{mL}$ )

\begin{tabular}{|c|c|c|c|c|c|c|c|}
\hline \multirow[t]{2}{*}{ Compounds } & \multicolumn{2}{|l|}{ G-negative } & \multicolumn{2}{|c|}{ G-positive } & \multicolumn{3}{|c|}{ Mycobacterium } \\
\hline & P. gingivalis & P. nigrescens & S. mitis & S. sanguinis & Tuberculosis & Avium & kansasii \\
\hline 4aa & $>200$ & $>200$ & $>200$ & $>200$ & $>2000$ & $>2000$ & $>2000$ \\
\hline $4 a b$ & 200 & 100 & $>200$ & $>200$ & $>2000$ & $>2000$ & $>2000$ \\
\hline $4 \mathbf{a c}$ & $>200$ & 200 & $>200$ & $>200$ & 500 & $>2000$ & $>2000$ \\
\hline 4ad & $>200$ & $>200$ & $>200$ & $>200$ & $>2000$ & $>2000$ & $>2000$ \\
\hline $4 \mathbf{a e}$ & $>200$ & 100 & 200 & $>200$ & $>2000$ & $>2000$ & 500 \\
\hline 4af & 200 & $>200$ & $>200$ & $>200$ & $>2000$ & $>2000$ & $>2000$ \\
\hline 4ag & $>200$ & $>200$ & $>200$ & $>200$ & $>2000$ & $>2000$ & $>2000$ \\
\hline 4ah & $>200$ & $>200$ & $>200$ & $>200$ & $>2000$ & $>2000$ & $>2000$ \\
\hline 4ai & $>200$ & $>200$ & $>200$ & $>200$ & $>2000$ & $>2000$ & $>2000$ \\
\hline 4aj & $>200$ & $>200$ & $>200$ & $>200$ & $>2000$ & $>2000$ & $>2000$ \\
\hline $4 a m$ & $>200$ & $>200$ & $>200$ & $>200$ & $>2000$ & $>2000$ & $>2000$ \\
\hline $4 a n$ & $>200$ & $>200$ & $>200$ & $>200$ & $>2000$ & $>2000$ & $>2000$ \\
\hline $4 \mathrm{ba}$ & 100 & $>200$ & 200 & 200 & 2000 & 2000 & 1000 \\
\hline $4 \mathrm{bb}$ & 50 & $>200$ & $>200$ & $>200$ & $>2000$ & $>2000$ & $>2000$ \\
\hline $4 b c$ & 200 & $>200$ & $>200$ & $>200$ & $>2000$ & $>2000$ & $>2000$ \\
\hline $4 \mathrm{bd}$ & 200 & $>200$ & $>200$ & $>200$ & $>2000$ & $>2000$ & $>2000$ \\
\hline $4 \mathrm{be}$ & $>200$ & $>200$ & $>200$ & $>200$ & $>2000$ & $>2000$ & $>2000$ \\
\hline $4 \mathrm{bf}$ & $>200$ & $>200$ & 200 & $>200$ & $>2000$ & $>2000$ & $>2000$ \\
\hline $4 \mathrm{bg}$ & 100 & $>200$ & $>200$ & $>200$ & $>2000$ & $>2000$ & $>2000$ \\
\hline $4 b h$ & $>200$ & $>200$ & $>200$ & $>200$ & $>2000$ & $>2000$ & $>2000$ \\
\hline $4 b i$ & 25 & $>200$ & $>200$ & 200 & 250 & 250 & 125 \\
\hline $4 \mathbf{b j}$ & $>200$ & $>200$ & $>200$ & $>200$ & $>2000$ & $>2000$ & $>2000$ \\
\hline $4 b n$ & 12,5 & $>200$ & $>200$ & $>200$ & $>2000$ & $>2000$ & $>2000$ \\
\hline $4 \mathrm{ca}$ & $>200$ & $>200$ & $>200$ & $>200$ & $>2000$ & $>2000$ & $>2000$ \\
\hline $4 \mathrm{cb}$ & $>200$ & $>200$ & $>200$ & $>200$ & $>2000$ & $>2000$ & $>2000$ \\
\hline $4 \mathrm{cc}$ & $>200$ & $>200$ & $>200$ & $>200$ & $>2000$ & $>2000$ & $>2000$ \\
\hline $4 \mathrm{~cd}$ & $>200$ & $>200$ & $>200$ & $>200$ & $>2000$ & $>2000$ & $>2000$ \\
\hline 4ce & 200 & $>200$ & $>200$ & $>200$ & $>2000$ & $>2000$ & $>2000$ \\
\hline $4 \mathrm{cf}$ & $>200$ & $>200$ & $>200$ & $>200$ & $>2000$ & $>2000$ & 500 \\
\hline $4 \mathrm{cg}$ & $>200$ & $>200$ & $>200$ & $>200$ & $>2000$ & $>2000$ & $>2000$ \\
\hline $4 \mathrm{ch}$ & 25 & $>200$ & $>200$ & $>200$ & $>2000$ & $>2000$ & $>2000$ \\
\hline $4 c i$ & 100 & $>200$ & $>200$ & $>200$ & $>2000$ & $>2000$ & $>2000$ \\
\hline $4 \mathrm{cn}$ & $>200$ & $>200$ & $>200$ & $>200$ & $>2000$ & $>2000$ & $>2000$ \\
\hline $4 \mathrm{~cm}$ & $>200$ & $>200$ & $>200$ & $>200$ & $>2000$ & $>2000$ & $>2000$ \\
\hline Isoniazid & - & - & - & - & 0.06 & $>1$ & 1 \\
\hline Chlorhexidine & 0.922 & 0.922 & 3.68 & 3.68 & - & - & - \\
\hline
\end{tabular}

-: not tested

Reactor Discover Reflux (CEM Corporation, $300 \mathrm{~W}$ ). Reactions were monitored using thin-layer chromatography (TLC) plates coated with $0.2 \mathrm{~mm}$ silica gel 60 F254 (Merck, Germany). TLC plates were visualized using ultraviolet (UV) irradiation $(254 \mathrm{~nm})$. The products were washed with hexane:ethyl acetate (8:2) for purification. Both ${ }^{1} \mathrm{H}$ and ${ }^{13} \mathrm{C}$ NMR spectra were determined on a Bruker ARX 400 spectrometer in DMSO- $d_{6}$. Proton chemical shifts in DMSO- $d_{6}$ are related to the middle of the residual multiplet $(\delta=2.50)$. Carbon chemical shifts were reported in parts per million $(\delta)$ relative to DMSO- $d_{6}$ (39.5 p.p.m.), and $\mathrm{J}$ (coupling constant) values were reported in hertz. The splitting patterns of protons are described as s (singlet), $d$ (doublet), dd (doublet of doublets), t (triplet) and $\mathrm{m}$ (multiplet). All melting points were determined on Melting Point B-540 Buchi apparatus and are uncorrected. The highresolution mass spectral data for new compounds were obtained using Bruker Daltonics-micrOTOF-Q, fitted with an ESI operating in the positive ion mode. 


\section{General experimental procedure and characterization of synthesized derivatives 4}

The mixture of tetronic acid $\mathbf{1}(1.0 \mathrm{mmol})$, aldehyde 2 (1.0 $\mathrm{mmol})$, and aniline $3(1.0 \mathrm{mmol})$ in $2 \mathrm{~mL}$ of $\mathrm{EtOH}$ was irradiated at a power of $200 \mathrm{~W}$ in an open vessel in a microwave reactor (reflux temperature of the solvent). The reaction was monitored using TLC every $3 \mathrm{~min}$ and after 15 min the reaction was cooled, the solvent was removed under vacuum and the solid obtained was washed with hexaneacetate $(8: 2)$.

\section{9-(benzo[d][1,3]dioxol-5-yl)-6,7,8-trimethoxy-4,9- dihydrofuro[3,4-b]quinolin-1(3H)-one (4aa)}

Yellow solid, yield 92\%, mp 271-273 ${ }^{\circ} \mathrm{C} .{ }^{1} \mathrm{H}$ NMR (400 MHz, DMSO- $d_{6}$ ): $\delta$ (p.p.m.) 9.93 (s, $\left.1 \mathrm{H}, \mathrm{NH}\right), 6.77$ (d, $1 \mathrm{H}$, $J=7.9 \mathrm{~Hz}), 6.63(\mathrm{~d}, 1 \mathrm{H}, J=1.7 \mathrm{~Hz}), 6.54(\mathrm{dd}, 1 \mathrm{H}, J=7.9$ and $1.7 \mathrm{~Hz}), 6.38(\mathrm{~s}, 1 \mathrm{H}), 5.94(\mathrm{~d}, 1 \mathrm{H}, J=0.9 \mathrm{~Hz}), 5.93(\mathrm{~d}$, $1 \mathrm{H}, J=0.9 \mathrm{~Hz}), 4.90(\mathrm{~d}, 1 \mathrm{H}, J=15.7 \mathrm{~Hz}), 4.88(\mathrm{~s}, 1 \mathrm{H})$, $4.79(\mathrm{~d}, 1 \mathrm{H}, J=15.7 \mathrm{~Hz}), 3.79(\mathrm{~s}, 3 \mathrm{H}), 3.64(\mathrm{~s}, 3 \mathrm{H}), 3.42$ (s, $3 \mathrm{H}) .{ }^{13} \mathrm{C}$ NMR $\left(100 \mathrm{MHz}, \mathrm{DMSO}-d_{6}\right): \delta$ (p.p.m.) 171.94, $157.59,152.77,151.66,146.84,145.27,141.11,137.47$, $132.99,120.37,110.05,108.00,107.69$, 95.92, 95.48, 64.84, 60.31, 60.013, 55.64, 34.92.

\section{9-(3,4-dimethoxyphenyl)-6,7,8-trimethoxy-4,9-dihydrofuro $[3,4-b]$ quinolin-1(3H)-one (4ab)}

White solid, yield 91\%, mp $258-260{ }^{\circ} \mathrm{C} .{ }^{1} \mathrm{H} \quad \mathrm{NMR}$ (400 MHz, DMSO-d $d_{6}$ ): $\delta$ (p.p.m.) 9.89 (s, 1H, NH), $6.80(\mathrm{~d}, 1 \mathrm{H}, J=8.2 \mathrm{~Hz}), 6.80(\mathrm{~d}, 1 \mathrm{H}, J=1.8 \mathrm{~Hz}), 6.51$ (dd, $1 \mathrm{H}, J=1.8$ and $8.2 \mathrm{~Hz}), 6.38(\mathrm{~s}, 1 \mathrm{H}), 4.90(\mathrm{~s}, 1 \mathrm{H})$, $4.88(\mathrm{~d}, 1 \mathrm{H}, J=15.7 \mathrm{~Hz}), 4.78(\mathrm{~d}, 1 \mathrm{H}, J=15.7 \mathrm{~Hz})$, $3.78(\mathrm{~s}, 3 \mathrm{H}), 3.68(\mathrm{~s}, 3 \mathrm{H}), 3.67(\mathrm{~s}, 3 \mathrm{H}), 3.63(\mathrm{~s}, 3 \mathrm{H})$, 3.39 (s, $3 \mathrm{H}),{ }^{13} \mathrm{C}$ NMR $\left(100 \mathrm{MHz}, \mathrm{DMSO}-d_{6}\right): \delta$ (p.p.m.) 172.12, 157.58, 152.70, 151.67, 148.10, 147.08, $139.68,137.52,133.02,119.42,111.76,111.66,10.13$, $99.11,95.53,64.87,64.83,60.32,59.98,55.69,55.46$, 34.70 .

\section{6,7,8-trimethoxy-9-(3,4,5-trimethoxyphenyl)-4,9- dihydrofuro[3,4-b]quinolin-1(3H)-one (4ac)}

Yellow solid, yield $89 \%$, mp $229-231^{\circ} \mathrm{C}$. ${ }^{1} \mathrm{H}$ NMR (400 MHz, DMSO- $d_{6}$ ): $\delta$ (p.p.m.) 9.91 (s, 1H, NH), 6.40 (s, 3H), $4.97(\mathrm{~s}, 1 \mathrm{H}), 4.92(\mathrm{~d}, 1 \mathrm{H}, J=15.6 \mathrm{~Hz}), 4.79$ (d, 1H, $J=$ $15.6 \mathrm{~Hz}), 3.80(\mathrm{~s}, 3 \mathrm{H}), 3.67(\mathrm{~s}, 3 \mathrm{H}), 3.65(\mathrm{~s}, 3 \mathrm{H}), 3.60(\mathrm{~s}$, $3 \mathrm{H}), 3.47(\mathrm{~s}, 3 \mathrm{H}), 3.46(\mathrm{~s}, 3 \mathrm{H}) .{ }^{13} \mathrm{C} \mathrm{NMR}(100 \mathrm{MHz}$, DMSO- $d_{6}$ ): $\delta$ (p.p.m.) 172.06, 157.87, 152.78, 152.40, $151.73,142.48,136.45,135.88,133.15,109.53,104.85$, $95.72,95.50,64.83,60.32,60.02,59.91,55.74,55.66$, 35.33 . 9-(4-chlorophenyl)-6,7,8-trimethoxy-4,9-dihydrofuro[3,4-b] quinolin-1(3H)-one (4ad)

Yellow solid, yield $81 \%, \mathrm{mp}>300{ }^{\circ} \mathrm{C} .{ }^{1} \mathrm{H}$ NMR $(400 \mathrm{MHz}$, DMSO-d ${ }_{6}$ ): $\delta$ (p.p.m.) 9.99 (s, 1H, NH), 7.31 (d, 2H, $J=$ $8.4 \mathrm{~Hz}), 7.13(\mathrm{~d}, 2 \mathrm{H}, J=8.4 \mathrm{~Hz}), 6.39(\mathrm{~s}, 1 \mathrm{H}), 4.96(\mathrm{~s}, 1 \mathrm{H})$, $4.90(\mathrm{~d}, 1 \mathrm{H}, J=15.7 \mathrm{~Hz}), 4.81(\mathrm{~d}, 1 \mathrm{H}, J=15.7 \mathrm{~Hz}), 3.79$ (s, 3H), 3.63 (s, 3H), 3.38 (s, 3H). ${ }^{13} \mathrm{C} \mathrm{NMR} \mathrm{(100} \mathrm{MHz,}$ DMSO- $\left.d_{6}\right): \delta$ (p.p.m.) $171.86,157.79,152.97,151.66$, $145.76,137.46,132.97,130.46,129.34,127.91,109.47$, 95.51, 95.37, 64.92, 60.30, 59.94, 55.63, 34.95. HRMS $\left(\mathrm{ESI}^{+}\right): \mathrm{m} / \mathrm{z}[\mathrm{M}+\mathrm{Na}]^{+}$calculated for $\mathrm{C}_{20} \mathrm{H}_{18} \mathrm{ClNO}_{5} \mathrm{Na}$ : 410.0764; found: 410.0755 .

9-(4-(benzyloxy)-3-methoxyphenyl)-6,7,8-trimethoxy-4,9dihydrofuro[3,4-b]quinolin-1(3H)-one (4ae)

White solid, yield $85 \%, \mathrm{mp}>300{ }^{\circ} \mathrm{C} .{ }^{1} \mathrm{H}$ NMR $(400 \mathrm{MHz}$, DMSO-d ) $\delta$ (p.p.m.) 9.89 (s, 1H, NH), 7.43-7.28 (m, 5H), $6.88(\mathrm{~d}, 1 \mathrm{H}, J=8.3 \mathrm{~Hz}), 6.83(\mathrm{~d}, 1 \mathrm{H}, J=2.0 \mathrm{~Hz}), 6.49$ (dd, $1 \mathrm{H}, J=8.3$ and $2.0 \mathrm{~Hz}), 6.38(\mathrm{~s}, 1 \mathrm{H}), 5.00(\mathrm{~s}, 1 \mathrm{H}), 4.89(\mathrm{~d}$, $1 \mathrm{H}, J=16.0 \mathrm{~Hz}), 4.79(\mathrm{~s}, 1 \mathrm{H}, J=16.0 \mathrm{~Hz}), 3.79(\mathrm{~s}, 3 \mathrm{H})$, 3.71 (s, 3H, 3.64 (s, 3H), 3.38 (s, 3H). ${ }^{13} \mathrm{C} \mathrm{NMR}(100 \mathrm{MHz}$, DMSO- $\left.d_{6}\right): \delta$ (p.p.m.) 172.03, 157.55, 152.70, 151.69, $148.53,146.01,140.18,137.48,137.23,133.06,128.31$, $127.70,119.38,113.56,112.01,110.07,96.03,95.47$, $69.97,64.81,60.30,59.94,55.65,55.53,34.78$. HRMS $\left(\mathrm{ESI}^{+}\right): \mathrm{m} / \mathrm{z}[\mathrm{M}+\mathrm{H}]^{+}$calculated for $\mathrm{C}_{28} \mathrm{H}_{28} \mathrm{NO}_{7}: 490.1857$; found: 490.1849 .

9-(4-fluorophenyl)-6,7,8-trimethoxy-4,9-dihydrofuro[3,4-b] quinolin-1(3H)-one (4af)

White solid, yield $82 \%, \mathrm{mp}>300{ }^{\circ} \mathrm{C} .{ }^{1} \mathrm{H}$ NMR $(400 \mathrm{MHz}$, DMSO- $d_{6}$ ): $\delta$ (p.p.m.) 9.96 (s, 1H, NH), 7.17-7.11 (m, 2H), 7.10-7.03 (m, 2H), 6.39 (s, 1H), 4.96 (s, 1H), 4.89 (d, 1H, $J=15.7 \mathrm{~Hz}), 4.81(\mathrm{~d}, 1 \mathrm{H}, J=15.7 \mathrm{~Hz}), 3.79(\mathrm{~s}, 3 \mathrm{H}), 3.63$ $(\mathrm{s}, 3 \mathrm{H}), 3.36(\mathrm{~s}, 3 \mathrm{H}) .{ }^{13} \mathrm{C}$ NMR $\left(100 \mathrm{MHz}, \mathrm{DMSO}-d_{6}\right): \delta$ (p. p.m.) 171.93, 161.69, 159.28, 157.70, 152.89, 151.66, $143.11,143.08,137.49,132.95,129.26,129.18,114.71$, 114.50, 109.82, 95.67, 95.51, 64.89, 60.29, 59.91, 55.65, 34.71. HRMS $\left(\mathrm{ESI}^{+}\right): \mathrm{m} / \mathrm{z} \quad[\mathrm{M}+\mathrm{H}]^{+}$calculated for $\mathrm{C}_{20} \mathrm{H}_{19} \mathrm{FNO}_{5}$ : 372.1241 ; found: 372.1244 .

6,7,8-trimethoxy-9-(4-(methylthio)phenyl)-4,9-dihydrofuro [3,4-b]quinolin-1(3H)-one (4ag)

White solid, yield $81 \%, \mathrm{mp} 221-223{ }^{\circ} \mathrm{C} .{ }^{1} \mathrm{H}$ NMR (400 MHz, DMSO- $d_{6}$ ): $\delta$ (p.p.m.) 9.94 (s, 1H, NH), 7.14 (d, 2H, $J=8.4 \mathrm{~Hz}), 7.06(\mathrm{~d}, 2 \mathrm{H}, J=8.4 \mathrm{~Hz}), 6.39$ (s, 1H), 4.91 (s, $1 \mathrm{H}), 4.89(\mathrm{~d}, 1 \mathrm{H}, J=15.8 \mathrm{~Hz}), 4.80(\mathrm{~d}, 1 \mathrm{H}, J=15.8 \mathrm{~Hz})$, 3.79 (s, 3H), 3.63 (s, 3H), 3.39 (s, 3H), $2.41(\mathrm{~s}, 3 \mathrm{H}) .{ }^{13} \mathrm{C}$ NMR (100 MHz, DMSO- $\left.d_{6}\right): \delta$ (p.p.m.) 171.91, 157.64, 
$152.81,151.67,143.76,137.46,135.24,133.02,128.09$, $125.75,109.88,95.75,95.49,64.86,60.30,59.96,55.64$, 34.87, 14.81. HRMS $\left(\mathrm{ESI}^{+}\right): \mathrm{m} / \mathrm{z}[\mathrm{M}+\mathrm{H}]^{+}$calculated for $\mathrm{C}_{21} \mathrm{H}_{22} \mathrm{SNO}_{5}$ : 400.1211; found: 400.1182 .

\section{6,7,8-trimethoxy-9-(4-(trifluoromethyl)phenyl)-4,9- dihydrofuro[3,4-b]quinolin-1(3H)-one (4ah)}

White solid, yield $84 \%, \mathrm{mp}>300{ }^{\circ} \mathrm{C} .{ }^{1} \mathrm{H}$ NMR $(400 \mathrm{MHz}$, DMSO- $d_{6}$ ): $\delta$ (p.p.m.) 10.05 (s, $\left.1 \mathrm{H}, \mathrm{NH}\right), 7.63$ (d, $1 \mathrm{H}, J=$ $8.2 \mathrm{~Hz}), 7.34$ (d, 1H, J=8.2 Hz), 6.41 (s, 1H), 5.07 (s, 1H), $4.91(\mathrm{~d}, 1 \mathrm{H}, J=15.8 \mathrm{~Hz}), 4.83(\mathrm{~d}, 1 \mathrm{H}, J=15.8 \mathrm{~Hz}), 3.80$ $(\mathrm{s}, 3 \mathrm{H}), 3.62(\mathrm{~s}, 3 \mathrm{H}), 3.77(\mathrm{~s}, 3 \mathrm{H}) .{ }^{13} \mathrm{C} \mathrm{NMR}(100 \mathrm{MHz}$, DMSO- $d_{6}$ ): $\delta$ (p.p.m.) 171.82, 157.99, 153.12, 151.65, $151.13,137.46,133.01,128.31,124.96,124.93,109.07$, 95.57, 95.02, 64.98, 60.31, 59.89, 55.67, 35.54. HRMS $\left(\mathrm{ESI}^{+}\right): \mathrm{m} / \mathrm{z} \quad[\mathrm{M}+\mathrm{H}]^{+}$calculated for $\mathrm{C}_{21} \mathrm{H}_{19} \mathrm{~F}_{3} \mathrm{NO}_{5}$ : 422.1209; found: 422.1208 .

\section{9-(4,5-dimethoxy-2-nitrophenyl)-6,7,8-trimethoxy-4,9- dihydrofuro[3,4-b]quinolin-1(3H)-one (4ai)}

Yellow solid, yield 76\%, mp 284-286 ${ }^{\circ} \mathrm{C} .{ }^{1} \mathrm{H}$ NMR (400 MHz, DMSO- $d_{6}$ ): $\delta$ (p.p.m.) 9.99 (s, 1H, NH), 7.45 (s, 1H), $6.57(\mathrm{~s}, 1 \mathrm{H}), 6.37(\mathrm{~s}, 1 \mathrm{H}), 6.00(\mathrm{~s}, 1 \mathrm{H}), 4.92(\mathrm{~d}, 1 \mathrm{H}, J=15.8$ $\mathrm{Hz}), 4.83$ (d, 1H, J=15.8 Hz), 3.81 (s, 3H), 3.78 (s, 3H), 3.65 (s, 3H), 3.60 (s, 3H), 3.40 (s, 3H). ${ }^{13} \mathrm{C} \mathrm{NMR}(100 \mathrm{MHz}$, DMSO- $d_{6}$ ): $\delta$ (p.p.m.) 171.50, 158.33, 153.13, 152.42, $151.63,146.65,140.13,137.32,135.28,132.87,112.495$, $109.25,107.24,95.63,94.65,64.96,60.34,59.91,55.92$, 55.72, 55.68, 30.00. HRMS $\left(\mathrm{ESI}^{+}\right): \mathrm{m} / \mathrm{z}\left[\mathrm{M}+\mathrm{H}-\mathrm{H}_{2} \mathrm{O}\right]^{+}$ calculated for $\mathrm{C}_{22} \mathrm{H}_{21} \mathrm{~N}_{2} \mathrm{O}_{8}$ : 441.1290; found: 441,1283

\section{9-(4-hydroxy-3-methoxyphenyl)-6,7,8-trimethoxy-4,9- dihydrofuro[3,4-b]quinolin-1(3H)-one (4aj)}

White solid, yield $80 \%$, mp $216-218{ }^{\circ} \mathrm{C} .{ }^{1} \mathrm{H}$ NMR (400 MHz, DMSO- $d_{6}$ ): $\delta$ (p.p.m.) 9.86 (s, 1H, NH), 8.72 (brs, $1 \mathrm{H}, \mathrm{OH}), 6.77(\mathrm{~d}, 1 \mathrm{H}, J=1.9 \mathrm{~Hz}), 6.62(\mathrm{~d}, 1 \mathrm{H}, J=8.1 \mathrm{~Hz})$, $6.41(\mathrm{dd}, 1 \mathrm{H}, J=8.1$ and $1.9 \mathrm{~Hz}), 6.37(\mathrm{~s}, 1 \mathrm{H}), 4.89(\mathrm{~d}, 1 \mathrm{H}$, $J=15.7 \mathrm{~Hz}), 4.87(\mathrm{~s}, 1 \mathrm{H}), 4.78(\mathrm{~d}, 1 \mathrm{H}, J=15.7 \mathrm{~Hz}), 3.79$ $(\mathrm{s}, 3 \mathrm{H}), 3.70$ (s, 3H), 3.64 (s, 3H), 3.39 (s, 3H). ${ }^{13} \mathrm{C} \mathrm{NMR}$ (100 MHz, DMSO- $d_{6}$ ): $\delta$ (p.p.m.) $172.06,157.44,152.61$, $151.70,146.90,144.71,138.25,137.50,133.05,119.75$, $115.09,112.12,110.39,96.29,95.48,64.78, .28,59.95$, 55.66, 55.63, 34.67. HRMS $\left(\mathrm{ESI}^{+}\right): \mathrm{m} / \mathrm{z}[\mathrm{M}+\mathrm{Na}]^{+}$calculated for $\mathrm{C}_{21} \mathrm{H}_{21} \mathrm{NO}_{7} \mathrm{Na}$ : 422.1208; found: 422.1212 .

\section{9-(3-hydroxyphenyl)-6,7,8-trimethoxy-4,9-dihydrofuro[3,4- b]quinolin-1(3H)-one (4ak)}

White solid, yield $81 \%$, mp $263-265{ }^{\circ} \mathrm{C}$. ${ }^{1} \mathrm{H}$ NMR (400 MHz, DMSO- $d_{6}$ ): $\delta$ (p.p.m.) 9.90 (s, 1H, NH), 9.19 (s, 1H,
$\mathrm{OH}), 7.01(\mathrm{t}, 1 \mathrm{H}, J=7.6 \mathrm{~Hz}), 6.56(\mathrm{~d}, 1 \mathrm{H}, J=7.6 \mathrm{~Hz})$, 6.54-6.48 (m, 2H), $6.38(\mathrm{~s}, 1 \mathrm{H}), 4.88(\mathrm{~d}, 1 \mathrm{H}, J=15.7 \mathrm{~Hz})$, $4.86(\mathrm{~s}, 1 \mathrm{H}), 4.79(\mathrm{~d}, 1 \mathrm{H}, J=15.7 \mathrm{~Hz}), 3.79(\mathrm{~s}, 3 \mathrm{H}), 3.64(\mathrm{~s}$, $3 \mathrm{H}), 3.37$ (s, 3H). ${ }^{13} \mathrm{C}$ NMR (100 MHz, DMSO- $\left.d_{6}\right): \delta$ (p.p. m.) $171.88,157.58,156.98,152.72,151.70,148.25$, $137.53,133.13,128.71,118.31,114.56,112.95,110.14$, $96.00,95.52,64.79,60.30,59.88,55.68,35.22$. HRMS $\left(\mathrm{ESI}^{+}\right): \mathrm{m} / \mathrm{z}[\mathrm{M}+\mathrm{H}]^{+}$calculated for $\mathrm{C}_{20} \mathrm{H}_{20} \mathrm{NO}_{6}: 370.1284$; found: 370.1286 .

6,7,8-trimethoxy-9-(3-methoxyphenyl)-4,9-dihydrofuro[3,4b]quinolin-1(3H)-one (4al)

White solid, yield 92.0\%, mp 231-233 ${ }^{\circ} \mathrm{C} .{ }^{1} \mathrm{H}$ NMR (400 MHz, DMSO- $d_{6}$ ): $\delta$ (p.p.m.) 9.93 (s, 1H, NH), 7.15 (t, $1 \mathrm{H}$, $J=8.2 \mathrm{~Hz}), 6.73-6.69$ (m, 1H), 6.67 (brd, $1 \mathrm{H}, J=1.4 \mathrm{~Hz})$, 6.68-6.65 (m, 2H), 6.39 (s, 1H), 4.93 (s, 1H), 4.89 (d, 1H, $J=15.8 \mathrm{~Hz}), 4.79(\mathrm{~d}, 1 \mathrm{H}, J=15.8 \mathrm{~Hz}), 3.79(\mathrm{~s}, 3 \mathrm{H}), 3.69$ (s, 3H), $3.36(\mathrm{~s}, 3 \mathrm{H}), 3.38(\mathrm{~s}, 3 \mathrm{H}) .{ }^{13} \mathrm{C} \mathrm{NMR}(100 \mathrm{MHz}$, DMSO- $d_{6}$ ): $\delta$ (p.p.m.) 171.89, 158.92, 157.73, 152.80, $151.67,148.35,137.50,133.12,128.95,119.82,113.85$, 110.67, 109.84, 95.81, 95.54, 64.83, 60.31, 59.91, 55.68, 54.83, 35.26. HRMS (ESI $\left.{ }^{+}\right): \mathrm{m} / \mathrm{z}[\mathrm{M}+\mathrm{H}]^{+}$calculated for $\mathrm{C}_{21} \mathrm{H}_{22} \mathrm{NO}_{6}$ : 384.1440; found: 384.1441 .

\section{9-(3,4-dihydroxyphenyl)-6,7,8-trimethoxy-4,9-dihydrofuro [3,4-b]quinolin-1(3H)-one (4am)}

White solid, yield $83 \%$, mp $272-274{ }^{\circ} \mathrm{C} .{ }^{1} \mathrm{H}$ NMR (400 MHz, DMSO- $d_{6}$ ): $\delta$ (p.p.m.) 9.84 (s, $\left.1 \mathrm{H}, \mathrm{NH}\right), 8.70$ (s, $1 \mathrm{H}$, $\mathrm{OH}), 8.59$ (s, 1H, OH), 6.57 (d, 1H, J=8.0 Hz), 6.50 (d, $1 \mathrm{H}, J=2.1 \mathrm{~Hz}), 6.38(\mathrm{dd}, 1 \mathrm{H}, \mathrm{J}=8,0$ and $2.1 \mathrm{~Hz}), 6.36(\mathrm{~s}$, $1 \mathrm{H}), 4.86(\mathrm{~d}, 1 \mathrm{H}, J=15.8 \mathrm{~Hz}), 4.77(\mathrm{~d}, 1 \mathrm{H}, J=15.8 \mathrm{~Hz})$, 4.77 (s, 1H), 3.78 (s, 3H), $3.64(\mathrm{~s}, 3 \mathrm{H}),{ }^{13} \mathrm{C}$ NMR (100 MHz, DMSO-d $\mathrm{d}_{6}$ ): $\delta$ (p.p.m.) 171.97, 157.24, 152.53, $151.72,144.58,143.38,138.27,137.54,133.04,118.25$, $115.11,114.91,110.72,96.48,95.47,64.72,60.28,59.94$, 55.67, 34.48. HRMS (ESI $\left.{ }^{+}\right): \mathrm{m} / \mathrm{z}[\mathrm{M}+\mathrm{H}]^{+}$calculated for $\mathrm{C}_{20} \mathrm{H}_{20} \mathrm{NO}_{7}$ : 386.1233; found: 386.1221 .

\section{6,7,8-trimethoxy-9-(6-nitrobenzo[d][1,3]dioxol-5-yl)-4,9- dihydrofuro[3,4-b]quinolin-1(3H)-one (4an)}

Orange solid, yield $70 \%$, mp $265-266{ }^{\circ} \mathrm{C} .{ }^{1} \mathrm{H}$ NMR (400 MHz, DMSO-d $d_{6}$ ): $\delta$ (p.p.m.) 10.03 (s, 1H, NH), 7.48 (s, $1 \mathrm{H}), 6.57(\mathrm{~s}, 1 \mathrm{H}), 6.37(\mathrm{~s}, 1 \mathrm{H}), 6.12(\mathrm{~d}, 2 \mathrm{H}, J=4.7 \mathrm{~Hz})$, $5.94(\mathrm{~s}, 1 \mathrm{H}), 4.93$ (d, 1H, $J=15.7), 4.83(\mathrm{~d}, 1 \mathrm{H}, J=15.7)$, 3.79 (s, 3H), $3.61(\mathrm{~s}, 3 \mathrm{H}), 3.44$ (s, 3H). ${ }^{13} \mathrm{C}$ NMR (100 $\left.\mathrm{MHz}, \mathrm{DMSO}-d_{6}\right): \delta$ (p.p.m.) 171.35, 158.42, 153.24, $151.58,151.21,145.75,141.38,137.74,137.28,132.76$, 109.18, 109.18, 104.17, 103.00, 95.71, 94.48, 65.00, 60.35, 59.92, 30.11. HRMS $\left(\mathrm{ESI}^{+}\right): \mathrm{m} / \mathrm{z}\left[\mathrm{M}+\mathrm{H}-\mathrm{H}_{2} \mathrm{O}\right]^{+}$calculated for $\mathrm{C}_{21} \mathrm{H}_{17} \mathrm{~N}_{2} \mathrm{O}_{8}$ : 425.0977: found: 425.0953 . 
9-(benzo[d][1,3]dioxol-5-yl)-6,9-dihydro-[1,3]dioxolo[4,5-g] furo[3,4-b]quinolin-8(5H)-one (4ba)

White solid, yield $86 \%, \mathrm{mp} 288-289^{\circ} \mathrm{C} .{ }^{1} \mathrm{H}$ NMR (400 MHz, DMSO-d $d_{6}$ ): $\delta$ (p.p.m.) 9.87 (s, 1H, NH), 6.78 (d, 1H, $J=8.0 \mathrm{~Hz}), 6.73(\mathrm{~s}, 1 \mathrm{H}), 6.65(\mathrm{~d}, 1 \mathrm{H}, J=8.0 \mathrm{~Hz}), 5.95$ (brs, 1H), 5.94 (brs, 1H), 5.93 (brs, 1H), 5.89 (brs, 1H), 4.94 (d, $1 \mathrm{H}, J=15.76 \mathrm{~Hz}), 4.83(\mathrm{~d}, 1 \mathrm{H}, J=15.7 \mathrm{~Hz}), 4.83$ (s, $1 \mathrm{H}){ }^{13} \mathrm{C}$ NMR $\left(100 \mathrm{MHz}, \mathrm{DMSO}-d_{6}\right): \delta$ (p.p.m.) 172.56 , $158.70,147.82,147.00,146.17,143.80,141.75,130.94$, $120.92,117.27,110.03,108.51,108.40,101.68,101.29$, $97.79,94.94,65.43,40.09$.

\section{9-(3,4-dimethoxyphenyl)-6,9-dihydro-[1,3]dioxolo[4,5-g] furo[3,4-b]quinolin-8(5H)-one (4bb)}

White solid, yield $86 \%, \mathrm{mp} 289-291{ }^{\circ} \mathrm{C} .{ }^{1} \mathrm{H}$ NMR (400 $\left.\mathrm{MHz}, \mathrm{DMSO}-d_{6}\right): \delta$ (p.p.m.) 9.84 (s, 1H, NH), 6.88 (s, 1H), $6.82(\mathrm{~d}, 1 \mathrm{H}, J=8.5 \mathrm{~Hz}), 6.62(\mathrm{brd}, 1 \mathrm{H}), 6.62$ (s, 1H), 6.52 (s, 1H), 5.91 (brs, 1H), 5.90 (brs, 1H), 4.96 $(\mathrm{d}, 1 \mathrm{H}, J=15.7 \mathrm{~Hz}), 4.85(\mathrm{~s}, 1 \mathrm{H}), 4,84(\mathrm{~d}, 1 \mathrm{H}, J=15.7$ $\mathrm{Hz}), 3.71$ (s, 3H), $3.69(\mathrm{~s}, 3 \mathrm{H}) .{ }^{13} \mathrm{C} \mathrm{NMR}(100 \mathrm{MHz}$, DMSO- $d_{6}$ ): $\delta$ (p.p.m.) 172.11, 158.12, 148.44, 147.25, $146.34,143.16,139,78,130.30,119.41,116.88,111.86$, $111.47,109,58,101.10,97.19,94.41,64.85,55.47,55.44$, 39.10 .

\section{9-(3,4,5-triimethoxy)-6,9-dihydro-[1,3]dioxolo[4,5-g]furo $[3,4-b] q u i n o l i n-8(5 H)$-one (4bc)}

White solid, yield 90\%, mp 271-274 ${ }^{\circ} \mathrm{C} .{ }^{1} \mathrm{H}$ NMR (400 MHz, DMSO- $d_{6}$ ): $\delta$ (p.p.m.) 9.86 (s, 1H, NH), 6.67 (s, 1H), $6.53(\mathrm{~s}, 1 \mathrm{H}), 6.48$ (s, 2H), $5.95(\mathrm{~d}, 1 \mathrm{H}, J=0.7 \mathrm{~Hz}), 5.90(\mathrm{~d}$, $1 \mathrm{H}, J=0.7 \mathrm{~Hz}), 4.98(\mathrm{~s}, 1 \mathrm{H}, J=15.7 \mathrm{~Hz}), 4.85(\mathrm{~s}, 1 \mathrm{H})$, $4.84(\mathrm{~d}, 1 \mathrm{H}, J=15.7 \mathrm{~Hz}), 3.70(\mathrm{~s}, 6 \mathrm{H}), 3.60(\mathrm{~s}, 3 \mathrm{H}) .{ }^{13} \mathrm{C}$ NMR (100 MHz, DMSO- $d_{6}$ ): $\delta$ (p.p.m.) 172.18, 158.41, $152.74,146.44,143.22,142.68,136.04,130.25,116.50$, 109.49, 104.82, 101.24, 101.14, 97.28, 94.14, 64.92, 59.86, $55.82,39.76$.

\section{9-(4-chlorophenyl)-6,9-dihydro-[1,3]dioxolo[4,5-g]furo[3,4- b]quinolin-8(5H)-one (4bd)}

White solid, yield $80 \%$, mp $290-292{ }^{\circ} \mathrm{C} .{ }^{1} \mathrm{H}$ NMR (400 MHz, DMSO-d $d_{6}$ ): $\delta$ (p.p.m.) 9.93 (s, 1H, NH), 7.33 (d, 2H, $J=8.4 \mathrm{~Hz}), 7.22(\mathrm{~d}, 2 \mathrm{H}, \quad J=8.4 \mathrm{~Hz}), \quad 6.58$ (s, 1H), 6.54 (s, 1H), 5.97 (brs, 1H), 5.91 (brs, 1H), 4.96 (s, 1H), $4.96(\mathrm{~d}, 1 \mathrm{H}, J=15.7 \mathrm{~Hz}), 4.86(\mathrm{~d}, 1 \mathrm{H}, J=15.7$ Hz). ${ }^{13} \mathrm{C}$ NMR (100 MHz, DMSO- $d_{6}$ ): $\delta$ (p.p.m.) 171.98, $158.37,146.61,145.72,143.36,130.90,130.48,129.34$, $128.24,116.05,109.53,101.22,97.35,93.92,64.97$, 38.95 . 9-(4-(benzyloxy)-3-methoxyphenyl)- 6,9-dihydro-[1,3] dioxolo[4,5-g]furo[3,4-b]quinolin-8(5H)-one (4be)

White solid, yield $84 \%, \mathrm{mp}>300{ }^{\circ} \mathrm{C} .{ }^{1} \mathrm{H}$ NMR $(400 \mathrm{MHz}$, DMSO-d $d_{6}$ ): $\delta$ (p.p.m.) 9.85 (s, 1H, NH), 7.45-7.29 (m, 5H), $6.92(\mathrm{~d}, 1 \mathrm{H}, J=1.9 \mathrm{~Hz}), 6.90(\mathrm{~d}, 1 \mathrm{H}, J=8.4 \mathrm{~Hz}), 6.63(\mathrm{~s}$, $1 \mathrm{H}), 6.59(\mathrm{dd}, 1 \mathrm{H}, J=8.4$ and $1.9 \mathrm{~Hz}), 6.52(\mathrm{~s}, 1 \mathrm{H}), 5.96$ (brs, 1H), 5.90 (brs, 1H), 5.01 (s, 2H), 4.96 (d, 1H, $J=15.7$ $\mathrm{Hz}), 4.85(\mathrm{~d}, 1 \mathrm{H}, J=15.7 \mathrm{~Hz}), 4.86(\mathrm{~s}, 1 \mathrm{H}) .{ }^{13} \mathrm{C}$ NMR $(100$ $\left.\mathrm{MHz}, \mathrm{DMSO}-d_{6}\right): \delta$ (p.p.m.) 172.12, 158.16, 148.76, $146.36,146,29,143.18,140.17,137.26,130.31,128.36$, $127.75,127.66,119.36,116.82,113.52,111.73,109.58$, 101.11, 97.20, 94.38, 69.908, 64.86, 55.53, 39.11. HRMS $\left(\mathrm{ESI}^{+}\right): \mathrm{m} / \mathrm{z}[\mathrm{M}+\mathrm{H}]^{+}$calculated for $\mathrm{C}_{26} \mathrm{H}_{22} \mathrm{NO}_{6}$ : 444.1440; found: 444.1444 .

9-(4-fluorphenyl)-6,9-dihydro-[1,3]dioxolo[4,5-g]furo[3,4-b] quinolin-8(5H)-one (4bf)

White solid, yield $81 \%, \mathrm{mp}>300{ }^{\circ} \mathrm{C} .{ }^{1} \mathrm{H}$ NMR $(400 \mathrm{MHz}$, DMSO- $d_{6}$ ): $\delta$ (p.p.m.) 9.90 (s, 1H, NH), 7.24-7.19 (m, 2H), 7.11-7.04 (m, 2H), $6.56(\mathrm{~s}, 1 \mathrm{H}), 6.53$ (s, 1H), 5.95 (brs, 1H), 5.90 (brs, 1H), 4.95 (s, 1H), $4.94(\mathrm{~d}, 1 \mathrm{H}, J=15.6 \mathrm{~Hz})$, $4.85(\mathrm{~d}, 1 \mathrm{H}, J=15.6 \mathrm{~Hz}) .{ }^{13} \mathrm{C}$ NMR $\left(100 \mathrm{MHz}, \mathrm{DMSO}-d_{6}\right)$ : $\delta$ (p.p.m.) $171.99,158.24,146.55,143.33,143.08,143.05$, $130.49,129.29,129.21,116.35,115.04,114.83,109.54$, 101.19, 97.31, 94.23, 64.93, 38.81. HRMS (ESI $\left.{ }^{+}\right): \mathrm{m} / \mathrm{z}[\mathrm{M}$ $+\mathrm{H}]^{+}$calculated for $\mathrm{C}_{18} \mathrm{H}_{13} \mathrm{FNO}_{4}$ : 326.0824; found: 326.0828 .

\section{9-(4-(methylthio)phenyl)-6,9-dihydro-[1,3]dioxolo[4,5-g]} furo[3,4-b]quinolin-8(5H)-one (4bg)

White solid, yield $81 \%$, mp $277-280{ }^{\circ} \mathrm{C} .{ }^{1} \mathrm{H}$ NMR (400 MHz, DMSO- $d_{6}$ ): $\delta$ (p.p.m.) 9.89 (s, 1H, NH), 7.18-7.12 $(\mathrm{m}, 4 \mathrm{H}), 6.56(\mathrm{~s}, 1 \mathrm{H}), 6.53(\mathrm{~s}, 1 \mathrm{H}), 5.96$ (brs, 1H), 5.90 (brs, 1H), 4.94 (d, 1H, J=15.6 Hz), 4.89 (s, 1H), 4.85 (d, $1 \mathrm{H}, J=15.6 \mathrm{~Hz}), 2.43(\mathrm{~s}, 3 \mathrm{H}) .{ }^{13} \mathrm{C} \mathrm{NMR}(100 \mathrm{MHz}$, DMSO- $\left.d_{6}\right): \delta$ (p.p.m.) 172.02, 158.22, 146.47, 143.75, $143.27,135.71,130.42,128.08,126.08,116.48,109.56$, 101.16, 97.27, 94.18, 64.91. HRMS (ESI $\left.{ }^{+}\right): \mathrm{m} / \mathrm{z}[\mathrm{M}+\mathrm{K}+$ $\left.\mathrm{H}_{2} \mathrm{O}-2 \mathrm{H}\right]^{+}$calculated for $\mathrm{C}_{19} \mathrm{H}_{16} \mathrm{NO}_{5} \mathrm{~S}$ : 408.0307; found: 408.0324 .

9-(4-(trifluoromethyl)phenyl)-6,9-dihydro-[1,3]dioxolo[4,5g]furo[3,4-b]quinolin-8(5H)-one (4bh)

White solid, yield $88 \%, \mathrm{mp}>300{ }^{\circ} \mathrm{C} .{ }^{1} \mathrm{H}$ NMR $(400 \mathrm{MHz}$, DMSO-d ) $\delta$ (p.p.m.) 9.98 (s, 1H, NH), 7.64 (d, 2H, $J=$ $8.2 \mathrm{~Hz}), 7.43(\mathrm{~d}, 2 \mathrm{H}, J=8.2 \mathrm{~Hz}), 6.58(\mathrm{~s}, 1 \mathrm{H}), 6.57(\mathrm{~s}, 1 \mathrm{H})$, 5.97 (brs, 1H), 5.91 (brs, 1H), 5.08 (s, 1H), 4.96 (d, 1H, J $=15.7 \mathrm{~Hz}), 4.88(\mathrm{~d}, 1 \mathrm{H}, J=15.7 \mathrm{~Hz}) .{ }^{13} \mathrm{C}$ NMR $(100$ 
MHz, DMSO- $\left.d_{6}\right): \delta$ (p.p.m.) 171.95, 158.54, 146.75, $143.45,130.57,128.35,125.29,125.26,115.53,109.54$, 101.27, 97.45, 93.70, 65.05, 39.47. HRMS (ESI $\left.{ }^{+}\right): \mathrm{m} / \mathrm{z}[\mathrm{M}$ $+\mathrm{Na}]^{+}$calculated for $\mathrm{C}_{19} \mathrm{H}_{13} \mathrm{~F}_{3} \mathrm{NO}_{4} \mathrm{Na}$ : 398.0611 ; found: 398.0609.

\section{9-(4,5-dimethoxy-2-nitrophenyl)-6,9-dihydro-[1,3]dioxolo [4,5-g]furo[3,4-b]quinolin-8(5H)-one (4bi)}

Yellow solid, yield 69\%, mp 243-244 ${ }^{\circ} \mathrm{C}$. ${ }^{1} \mathrm{H}$ NMR (400 $\left.\mathrm{MHz}, \mathrm{DMSO}-d_{6}\right): \delta$ (p.p.m.) 9.96 (s, 1H, NH), 7.43 $(\mathrm{s}, 1 \mathrm{H}), 6.71(\mathrm{~s}, 1 \mathrm{H}), 6.57(\mathrm{~s}, 1 \mathrm{H}), 5.97$ (brs, 1H), 5.94 (brs, 1H), 5.69 (s, 1H), $4.93(\mathrm{~d}, 1 \mathrm{H}, J=15.6 \mathrm{~Hz}), 4,84$ $(\mathrm{d}, 1 \mathrm{H}, J=15.6 \mathrm{~Hz}), 3.83(\mathrm{~s}, 3 \mathrm{H}), 3.71(\mathrm{~s}, 3 \mathrm{H}) .{ }^{13} \mathrm{C} \mathrm{NMR}$ (100 MHz, DMSO-d ${ }_{6}$ ): $\delta$ (p.p.m.) $171.65,158.39,152.68$, $147.14, \quad 146.87,143.56,140.69,134.45,130.86$, $115.11,112.95,108.86,107,10,101.34,97.63,93.76$, 64.99, 56.01, 55.89, 34.32. HRMS $\left(\mathrm{ESI}^{+}\right): \mathrm{m} / \mathrm{z}[\mathrm{M}+\mathrm{H}-$ $\mathrm{H}_{2} \mathrm{O}^{+}$calculated for $\mathrm{C}_{20} \mathrm{H}_{15} \mathrm{~N}_{2} \mathrm{O}_{7}$ : 395.0872; found: 395.0869 .

\section{9-(4-hydroxy-3-methoxyphenyl)-6,9-dihydro-[1,3]dioxolo [4,5-g]furo[3,4-b]quinolin-8(5H)-one (4bj)}

White solid, yield $81 \%$, mp 279-281 ${ }^{\circ} \mathrm{C} .{ }^{1} \mathrm{H}$ NMR (400 MHz, DMSO- $d_{6}$ ): $\delta$ (p.p.m.) 9.80 (s, 1H, NH), 8.76 (s, 1H, $\mathrm{OH}), 6.84(\mathrm{~d}, 1 \mathrm{H}, J=1.8 \mathrm{~Hz}), 6.64(\mathrm{~d}, 1 \mathrm{H}, J=8.0 \mathrm{~Hz})$, $6.61(\mathrm{~s}, 1 \mathrm{H}), 6.51(\mathrm{~s}, 1 \mathrm{H}), 6.49(\mathrm{dd}, 1 \mathrm{H}, J=1.8$ and 8.0 $\mathrm{Hz}$ ), 5.95 (brs, 1H), 5.90 (brs, 1H), 4.94 (d, 1H, $J=15.6$ $\mathrm{Hz}$, 4.83 (d, 1H, $J=15.6 \mathrm{~Hz}), 4.79$ (s, 1H), 3.3 (s, 3H). ${ }^{13} \mathrm{C}$ NMR (100 MHz, DMSO- $\left.d_{6}\right): \delta$ (p.p.m.) 172.12, $158.02,147.24,146.28,144.97,143.14,38.31,130.30$, $119.72, \quad 117.14, \quad 115.33,111.89,109.57,101.06$, 97.15, 94.61, 64.81, 55.63, 39.12. HRMS (ESI $\left.{ }^{+}\right): \mathrm{m} / \mathrm{z}[\mathrm{M}$ $+\mathrm{Na}]^{+}$calculated for $\mathrm{C}_{19} \mathrm{H}_{15} \mathrm{NO}_{6} \mathrm{Na}$ : 376.0791; found 376.0792

\section{9-(6-nitrobenzo[d][1,3]dioxol-5-yl)-6,9-dihydro-[1,3]dioxolo [4,5-g]furo[3,4-b]quinolin-8(5H)-one (4bn)}

Yellow solid, yield $71 \%$, mp $225-227{ }^{\circ} \mathrm{C} .{ }^{1} \mathrm{H}$ NMR (400 MHz, DMSO- $d_{6}$ ): $\delta$ (p.p.m.) 10.00 (s, 1H, NH), 7.47 (s, $1 \mathrm{H}), 6.71(\mathrm{~s}, 1 \mathrm{H}), 6.58(\mathrm{~s}, 2 \mathrm{H}), 6.16(\mathrm{~d}, 1 \mathrm{H}, J=0.8 \mathrm{~Hz})$, $6.14(\mathrm{~d}, 1 \mathrm{H}, J=0.8 \mathrm{~Hz}), 5.98(\mathrm{~d}, 1 \mathrm{H}, J=0.7 \mathrm{~Hz}), 5.94(\mathrm{~d}$, $1 \mathrm{H}, J=0.7 \mathrm{~Hz}), 5.62(\mathrm{~s}, 1 \mathrm{H}), 4.92(\mathrm{~d}, 1 \mathrm{H}, J=15.5 \mathrm{~Hz})$, $4.85(\mathrm{~d}, 1 \mathrm{H}, J=15.5 \mathrm{~Hz}) .{ }^{13} \mathrm{C}$ NMR $\left(100 \mathrm{MHz}, \mathrm{DMSO}-d_{6}\right)$ : $\delta$ (p.p.m.) 171.62, 158.38, 151.50, 147.00, 146.18, 143.66, 141.87, 136.94, 130.84, 114.89, 109.64, 108.85, 103.84, 103.16, 101.39, 97.68, 93.72, 68.08, 34.15. HRMS (ESI $\left.{ }^{+}\right)$: $\mathrm{m} / \mathrm{z} \quad\left[\mathrm{M}+\mathrm{H}-\mathrm{H}_{2} \mathrm{O}\right]^{+}$calculated for $\mathrm{C}_{19} \mathrm{H}_{11} \mathrm{~N}_{2} \mathrm{O}_{7}$ : 379.0560; found: 379.0558 9-(benzo[d][1,3]dioxol-5-yl)-6,7-dimethoxy-4,9-dihydrofuro [3,4-b]quinolin-1(3H)-one (4ca)

White solid, yield 91\%, mp $287-289^{\circ} \mathrm{C} .{ }^{1} \mathrm{H}$ NMR (400 MHz, DMSO-d $)_{6}$ ): $\delta$ (p.p.m.) 9.85 (s, 1H, NH), 6.79 (d, $1 \mathrm{H}$, $J=7.9 \mathrm{~Hz}), 6.73(\mathrm{~d}, 1 \mathrm{H}, J=1.6 \mathrm{~Hz}), 6.66(\mathrm{dd}, 1 \mathrm{H}, J=7.9$ and $1.6 \mathrm{~Hz}), 6.62(\mathrm{~s}, 1 \mathrm{H}), 6.51(\mathrm{~s}, 1 \mathrm{H}), 5.95(\mathrm{brs}, 1 \mathrm{H}), 5.94$ (brs, 1H), 4.95 (d, 1H, J=15.5 Hz), 4.87 (s, 1H), 4.84 (d, $1 \mathrm{H}, J=15.5 \mathrm{~Hz}), 3.73(\mathrm{~s}, 3 \mathrm{H}), 3.60(\mathrm{~s}, 3 \mathrm{H}) .{ }^{13} \mathrm{C}$ NMR $(100$ $\left.\mathrm{MHz}, \mathrm{DMSO}-d_{6}\right): \delta$ (p.p.m.) 172.15, 158.20, 148.16, 147.19 , 145.51, 144.92, 141.15, 129.63, 120.35, 115.44, $113.71,107.97,107.85,100.72,100.34,99.46,64.92$, $55.84,55.43,38.86$.

\section{9-(3,4-dimethoxyphenyl)-6,7-dimethoxy-4,9-dihydrofuro [3,4-b]quinolin-1(3H)-one (4cb)}

Yellow solid, yield 90\%, mp 228-230 ${ }^{\circ} \mathrm{C} .{ }^{1} \mathrm{H}$ NMR (400 MHz, DMSO-d $): \delta$ (p.p.m.) 9.82 (s, $1 \mathrm{H}, \mathrm{NH}), 6.89$ (d, $1 \mathrm{H}$, $J=1.9 \mathrm{~Hz}), 6.1(\mathrm{~d}, 1 \mathrm{H}, J=8.6 \mathrm{~Hz}), 6.66(\mathrm{~s}, 1 \mathrm{H}), 6.60(\mathrm{dd}$, $1 \mathrm{H}, J=8.3$ and $1.9 \mathrm{~Hz}), 6.56(\mathrm{~s}, 1 \mathrm{H}), 4.95(\mathrm{~d}, 1 \mathrm{H}, J=15.6$ $\mathrm{Hz}), 4.88$ (s, 1H), $4.84(\mathrm{~d}, 1 \mathrm{H}, J=15.6 \mathrm{~Hz}), 3.73$ (s, 3H), 3.71 (s, 3H), 3.69 (s, 3H), 3.60 (s, 3H). ${ }^{13} \mathrm{C}$ NMR (100 $\left.\mathrm{MHz}, \mathrm{DMSO}-d_{6}\right): \delta$ (p.p.m.) 172.22, 158.18, 148.40, $148.07,147.16,144.85,139.59,29.62,119.35,115.56$, $113.78,111.71,111.49,100.29,94.48,64.88,55.83,55.44$, $55.42,38.74$.

\section{6,7-dimethoxy-9-(3,4,5-trimethoxyphenyl)-4,9-dihydrofuro [3,4-b]quinolin-1(3H)-one (4cc)}

White solid, yield 92\%, mp $236-238^{\circ} \mathrm{C} .{ }^{1} \mathrm{H}$ NMR (400 MHz, DMSO-d $d_{6}$ ): $\delta$ (p.p.m.) 9.83 (s, 1H, NH), 6.85 (s, 1H), $6.73(\mathrm{~s}, 1 \mathrm{H}), 6.52(\mathrm{~s}, 1 \mathrm{H}), 6.50(\mathrm{~s}, 1 \mathrm{H}), 5.00(\mathrm{~d}, 1 \mathrm{H}, J=$ $15.5 \mathrm{~Hz}), 4.89$ (s, 1H), $4.85(\mathrm{~d}, 1 \mathrm{H}, J=15.5 \mathrm{~Hz}), 3.73(\mathrm{~s}$, 3H), $3.70(\mathrm{~s}, 3 \mathrm{H}), 3.62(\mathrm{~s}, 3 \mathrm{H}), 3.61(\mathrm{~s}, 3 \mathrm{H}) .{ }^{13} \mathrm{C}$ NMR $(100$ MHz, DMSO- $d_{6}$ ): $\delta$ (p.p.m.) 17.22, 158.51, 15.70, 148.20, $144.87,142.49,135.95,129.63,115.17,113.83,107.62$, 104.78, 100.78, 94.11, 64.94, 59.85, 56.08, 55.99, 55.93, $55.8,55.45,18.52$.

\section{9-(4-chlorophenyl)-6,7-dimethoxy-4,9-dihydrofuro[3,4-b] quinolin-1(3H)-one $(4 \mathrm{~cd})$}

White solid, yield $81 \%, \mathrm{mp} 265-267{ }^{\circ} \mathrm{C} .{ }^{1} \mathrm{H}$ NMR (400 MHz, DMSO- $d_{6}$ ): $\delta$ (p.p.m.) 9.88 (s, $\left.1 \mathrm{H}, \mathrm{NH}\right), 7.31$ (d, 2H, $J=8.4 \mathrm{~Hz}), 7.21(\mathrm{~d}, 2 \mathrm{H}, J=8.4 \mathrm{~Hz}), 6.58(\mathrm{~s}, 1 \mathrm{H}), 6.52$ (s, $1 \mathrm{H}), 4.97$ (s, 1H), 4.94 (d, 1H, $J=15.7 \mathrm{~Hz}), 4.85$ (d, 1H, $J$ $=15.7 \mathrm{~Hz}), 3.73(\mathrm{~s}, 3 \mathrm{H}), 3.57(\mathrm{~s}, 3 \mathrm{H}) .{ }^{13} \mathrm{C} \mathrm{NMR}(100 \mathrm{MHz}$, DMSO- $d_{6}$ ): $\delta$ (p.p.m.) 172.04, 158.34, 148.37, 145.63, $145.08,130.78,129.79,129.35,128.18,114.78,113.83$, 
100.52, 94.09, 64.99, 55.89, 55.48, 38.66. HRMS (ESI $\left.{ }^{+}\right)$: $\mathrm{m} / \mathrm{z}[\mathrm{M}+\mathrm{Na}]^{+}$calculated for $\mathrm{C}_{19} \mathrm{H}_{17} \mathrm{ClNO}_{4} \mathrm{Na}$ : 380.0659: found: 380.0661 .

\section{9-(4-(benzyloxy)-3-methoxyphenyl)-6,7-dimethoxy-4,9- dihydrofuro[3,4-b]quinolin-1(3H)-one (4ce)}

White solid, yield 92\%, mp 232-234 ${ }^{\circ} \mathrm{C}$. ${ }^{1} \mathrm{H}$ NMR (400 MHz, DMSO- $d_{6}$ ): $\delta$ (p.p.m.) 9.80 (s, $\left.1 \mathrm{H}, \mathrm{NH}\right), 7.44-7.28$ $(\mathrm{m}, 5 \mathrm{H}), 6.92(\mathrm{~d}, 1 \mathrm{H}, J=1.9 \mathrm{~Hz}), 6.89(\mathrm{~d}, 1 \mathrm{H}, J=8.3 \mathrm{~Hz})$, $6.66(\mathrm{~s}, 1 \mathrm{H}), 6.56(\mathrm{dd}, 1 \mathrm{H}, J=8.3$ and $1.9 \mathrm{~Hz}), 6.50(\mathrm{~s}, 1 \mathrm{H})$, 4.99 (s, 1H), 4.94 (d, 1H, $J=15.6 \mathrm{~Hz}), 4.87$ (s, 1H), 4.83 $(\mathrm{d}, 1 \mathrm{H}, J=15.6 \mathrm{~Hz}) .{ }^{13} \mathrm{C}$ NMR $\left(100 \mathrm{MHz}, \mathrm{DMSO}-d_{6}\right): \delta(\mathrm{p}$. p.m.) $172.95,158.19,148.81,148.16,146.27,144.92$, 140.02 , 137.27, 129.70, 128.34, 127.73, 127.68, 119.36, $115.56,113.93,113.56,111.89,100.41,94.50,69.98$, 64.89, 55.91, 55.61, 55.48, 38.76. HRMS (ESI $\left.{ }^{+}\right): \mathrm{m} / \mathrm{z}[\mathrm{M}$ $+\mathrm{Na}]^{+}$calculated for $\mathrm{C}_{27} \mathrm{H}_{25} \mathrm{NO}_{6} \mathrm{Na}$ : 482.1571; found: 482.1578 .

\section{9-(4-fluorophenyl)-6,7-dimethoxy-4,9-dihydrofuro[3,4-b] quinolin-1(3H)-one (4cf)}

White solid, yield $79 \%$, mp $257-258{ }^{\circ} \mathrm{C} .{ }^{1} \mathrm{H}$ NMR (400 $\mathrm{MHz}, \mathrm{DMSO}-d_{6}$ ): $\delta$ (p.p.m.) 9.87 (s, 1H, NH), 7.24-7.20 (m, 2H), 7.11-7.06 (m, 2H), 6.59 (s, 1H), 6.53 (s, 1H), 4.98 (s, 1H), $4.94(\mathrm{~d}, 1 \mathrm{H}, J=15.6 \mathrm{~Hz}), 4.85(\mathrm{~d}, 1 \mathrm{H}, J=15.6$ $\mathrm{Hz}), 3.73$ (s, 3H), $3.58(\mathrm{~s}, 3 \mathrm{H}) .{ }^{13} \mathrm{C} \mathrm{NMR}(100 \mathrm{MHz}$, DMSO- $d_{6}$ ): $\delta$ (p.p.m.) 172.08, 158.24, 1478.31, 145.05, $14.98,142.95,129.79,129.28,129.20,115.98,114.98$, 114.77, 113.90, 100.50, 94.35, 64.95, 55.90, 55.49, 38.50. HRMS $\left(\mathrm{ESI}^{+}\right): \mathrm{m} / \mathrm{z}[\mathrm{M}+\mathrm{H}]^{+}$calculated for $\mathrm{C}_{19} \mathrm{H}_{17} \mathrm{FNO}_{4}$ : 342.1136; found: 342.1133 .

\section{6,7,8-trimethoxy-9-(4-(methylthio)phenyl)-4,9-dihydrofuro [3,4-b]quinolin-1(3H)-one $(4 \mathrm{cg})$}

White solid, yield $81 \%$, mp $286-287{ }^{\circ} \mathrm{C} .{ }^{1} \mathrm{H}$ NMR (400 MHz, DMSO- $d_{6}$ ): $\delta$ (p.p.m.) 9.85 (s, 1H, NH), 7.17-7.12 $(\mathrm{m}, 4 \mathrm{H}), 6.58(\mathrm{~s}, 1 \mathrm{H}), 6.52(\mathrm{~s}, 1 \mathrm{H}), 4.93(\mathrm{~d}, 1 \mathrm{H}, J=15.7$ $\mathrm{Hz}), 4.91(\mathrm{~s}, 1 \mathrm{H}), 4.84(\mathrm{~d}, 1 \mathrm{H}, J=15.7 \mathrm{~Hz}), 3.73(\mathrm{~s}, 3 \mathrm{H})$, 3.58 (s, 3H), 2.42 (s, 3H). ${ }^{13} \mathrm{C}$ NMR (100 MHz, DMSO- $\left.d_{6}\right)$ : $\delta$ (p.p.m.) 172.13, 158.22, 148.25, 145.01, 143.65, 135.55, $129.74,128.08,126.03,115.25,113.86,100.47,94.37$, 64.94, 55.89, 55.44, 55.49, 38.74, 14.78. HRMS (ESI $\left.{ }^{+}\right): \mathrm{m} /$ $\mathrm{z}[\mathrm{M}+\mathrm{H}]^{+}$calculated for $\mathrm{C}_{20} \mathrm{H}_{20} \mathrm{NO}_{4} \mathrm{~S}$ : 370.1106; found: 370.1109 .

\section{6,7-dimethoxy-9-(4-(trifluoromethyl)phenyl)-4,9- dihydrofuro[3,4-b]quinolin-1(3H)-one $(4 \mathrm{ch})$}

White solid, yield $82 \%$, mp $255-256{ }^{\circ} \mathrm{C}$. ${ }^{1} \mathrm{H}$ NMR (400 MHz, DMSO- $d_{6}$ ): $\delta$ (p.p.m.) 9.95 (s, 1H, NH), 7.64 (d, 2H,
$J=8.1 \mathrm{~Hz}), 7.43(\mathrm{~d}, 2 \mathrm{H}, J=8.1 \mathrm{~Hz}), 6.60(\mathrm{~s}, 1 \mathrm{H}), 6.56(\mathrm{~s}$, $1 \mathrm{H}), 5.10(\mathrm{~s}, 1 \mathrm{H}), 4.97(\mathrm{~d}, 1 \mathrm{H}, J=15.7 \mathrm{~Hz}), 4.87(\mathrm{~d}, 1 \mathrm{H}, J$ $=15.7 \mathrm{~Hz}), 3.75(\mathrm{~s}, 3 \mathrm{H}), 3.58(\mathrm{~s}, 3 \mathrm{H}) .{ }^{13} \mathrm{C} \mathrm{NMR}(100 \mathrm{MHz}$, DMSO- $\left.d_{6}\right): \delta$ (p.p.m.) 172.02, 158.53, 150.96, 148.49, $145.16,130.67,129.88,128.33,125.21,125.18,114.30$, 113.83, 100.61, 93.85, 65.07, 55.88, 55.48, 39.15. HRMS $\left(\mathrm{ESI}^{+}\right): \mathrm{m} / \mathrm{z} \quad[\mathrm{M}+\mathrm{H}]^{+}$calculated for $\mathrm{C}_{20} \mathrm{H}_{17} \mathrm{~F}_{3} \mathrm{NO}_{4}$ : 392.1104; found: 392.1105 .

\section{9-(4,5-dimethoxy-2-nitrophenyl)-6,7-dimethoxy-4,9- dihydrofuro[3,4-b]quinolin-1(3H)-one (4ci)}

Yellow solid, yield 77\%, mp 249-251 ${ }^{\circ} \mathrm{C} .{ }^{1} \mathrm{H}$ NMR (400 MHz, DMSO- $d_{6}$ ): $\delta$ (p.p.m.) 9.94 (s, 1H, NH), 7.45 $(\mathrm{s}, 1 \mathrm{H}), 6.68(\mathrm{~s}, 1 \mathrm{H}), 6.65(\mathrm{~s}, 1 \mathrm{H}), 6.57(\mathrm{~s}, 1 \mathrm{H}), 5.73(\mathrm{~s}$, $1 \mathrm{H}), 4.95(\mathrm{~d}, 1 \mathrm{H}, J=15.6 \mathrm{~Hz}), 4.85(\mathrm{~d}, 1 \mathrm{H}, J=15.6 \mathrm{~Hz})$, $3.83(\mathrm{~s}, 3 \mathrm{H}), 3.76(\mathrm{~s}, 3 \mathrm{H}), 3.70(\mathrm{~s}, 3 \mathrm{H}), 3.57(\mathrm{~s}, 3 \mathrm{H}) .{ }^{13} \mathrm{C}$ NMR (100 MHz, DMSO- $\left.d_{6}\right): \delta$ (p.p.m.) 171.77, 158.48, $152.61,148.53,147.01,145.11,140.69,134.52,130.04$, $113.93,112.98,112.75,107.00,100.63,93.87,65.04$, 55.95, 55.83, 55.69, 55.42, 33.81. HRMS $\left(\mathrm{ESI}^{+}\right): \mathrm{m} / \mathrm{z}$ $[\mathrm{M}+\mathrm{H}]^{+}$calculated for $\mathrm{C}_{21} \mathrm{H}_{21} \mathrm{~N}_{2} \mathrm{O}_{8}$ : 429.1290: found: 429.1297.

\section{9-(3,4-dihydroxyphenyl)-6,7-dimethoxy-4,9-dihydrofuro [3,4-b]quinolin-1(3H)-one $(4 \mathrm{~cm})$}

White solid, yield $80 \%, \mathrm{mp} 244-246{ }^{\circ} \mathrm{C} .{ }^{1} \mathrm{H}$ NMR (400 MHz, DMSO- $d_{6}$ ): $\delta$ (p.p.m.) 9.75 (s, $\left.1 \mathrm{H}, \mathrm{NH}\right), 8.70(\mathrm{~s}, 1 \mathrm{H}$, $\mathrm{OH}), 8.64(\mathrm{~s}, 1 \mathrm{H}, \mathrm{OH}), 6.58(\mathrm{~d}, 1 \mathrm{H}, J=8.0 \mathrm{HZ}), 6.55$ $(\mathrm{s}, 1 \mathrm{H}), 6.50(\mathrm{~d}, 1 \mathrm{H}, J=2.0 \mathrm{~Hz}), 6.49(\mathrm{~s}, 1 \mathrm{H}), 6.46(\mathrm{dd}$, $1 \mathrm{H}, J=8.0$ and $2.0 \mathrm{~Hz}), 4.89(\mathrm{~d}, 1 \mathrm{H}, J=15.7 \mathrm{~Hz}), 4.81$ $(\mathrm{d}, 1 \mathrm{H}, J=15.7 \mathrm{~Hz}), 4.71(\mathrm{~s}, 1 \mathrm{H}), 3.72(\mathrm{~s}, 3 \mathrm{H}), 3.58(\mathrm{~s}$, $3 \mathrm{H}) .{ }^{13} \mathrm{C}$ NMR (100 MHz, DMSO- $d_{6}$ ): $\delta$ (p.p.m.) 172.14 , $157.76,148.06,144.92,144.89,143.63,138.27,129.70$, $118.19,116.06,115.10,115.03,113.95,100.35,94.98$, 64.73, 55.87, 55.49, 38.67. HRMS $\left(\mathrm{ESI}^{+}\right): \mathrm{m} / \mathrm{z}[\mathrm{M}+\mathrm{H}+$ $\mathrm{K}]^{+}$calculated for $\mathrm{C}_{19} \mathrm{H}_{18} \mathrm{NO}_{6} \mathrm{~K}:$ 395.0765; found: 395.0761

\section{6,7-dimethoxy-9-(6-nitrobenzo[d][1,3]dioxol-5-yl)-4,9- dihydrofuro[3,4-b]quinolin-1(3H)-one (4cn)}

Yellow solid, yield $70 \%$, mp $245-246{ }^{\circ} \mathrm{C} .{ }^{1} \mathrm{H}$ NMR (400 MHz, DMSO- $d_{6}$ ): $\delta$ (p.p.m.) 9.98 (s, 1H, NH), 6.67 (s, 1H), $6.65(\mathrm{~s}, 1 \mathrm{H}), 6.56(\mathrm{~s}, 1 \mathrm{H}), 6.15(\mathrm{~s}, 1 \mathrm{H}), 6.14(\mathrm{~s}, 1 \mathrm{H}), 5.64(\mathrm{~s}$, $1 \mathrm{H}), 4.94(\mathrm{~d}, 1 \mathrm{H}, J=15.6 \mathrm{~Hz}), 4.87(\mathrm{~d}, 1 \mathrm{H}, J=15.6 \mathrm{~Hz})$, 3.75 (s, 3H), 3.58(s, 3H). ${ }^{13} \mathrm{C}$ NMR (100 MHz, DMSO- $\left.d_{6}\right)$ : $\delta$ (p.p.m.) 171.74, 158.50, 141.45, 148.63, 146.08, 145.21, $141.84,137.00,129.97,113.75,112.95,109.53,103.88$, $103.14,100.71,93.78,65.13,55.69,55.45,33.73$. HRMS $\left(\mathrm{ESI}^{+}\right): \mathrm{m} / \mathrm{z}\left[\mathrm{M}+\mathrm{H}-\mathrm{H}_{2} \mathrm{O}\right]^{+}$calculated for $\mathrm{C}_{20} \mathrm{H}_{15} \mathrm{~N}_{2} \mathrm{O}_{7}$ : 395.0878; found: 395.0872 . 


\section{Biological activity}

The antibacterial activity of the derivatives $\mathbf{4}$ was assessed against Porphyromonas gingivalis (ATCC 33277), Prevotella nigrescens (ATCC 33563), Streptococcus mitis (ATCC 49456), Streptococcus sanguinis (ATCC 10556), Mycobacterium tuberculosis (ATCC 27294), Mycobacterium avium (ATCC 25291), and Mycobacterium kansasii (ATCC 12478).

The minimal inhibitory concentration (MIC) of the derivatives $\mathbf{4}$ was determined using the microdilution broth method in 96-well microplates (Sousa et al. 2015). Samples were dissolved in dimethyl sulfoxide (DMSO) to a concentration of $8000 \mu \mathrm{g} / \mathrm{mL}$, followed by dilution in tryptic soy broth (TSB) for aerobic microorganisms and Schaedler broth (Difco) supplemented which hemin $(5.0 \mathrm{~g} / \mathrm{mL})$, and menadione $(10.0 \mathrm{~g} / \mathrm{mL})$ for anaerobic microorganisms; the sample concentrations tested ranged from 200 to $0.195 \mu \mathrm{g} /$ $\mathrm{mL}$. The final DMSO content was $4 \%(\mathrm{v} / \mathrm{v})$, and this solution was used as a negative control. The inoculum was adjusted for each organism to yield a cell concentration of $5 \times 10^{5}$ colony forming units per $\mathrm{mL}(\mathrm{CFU} / \mathrm{mL})$, according to the Clinical and Laboratory Standards Institute (CLSI 2012a) guidelines (CLSI 2012b). The 96-well microplates containing the aerobic microorganisms were closed with a sterile plate sealer and incubated aerobically at $37^{\circ} \mathrm{C}$ for 24 $\mathrm{h}$. The plates containing the anaerobic microorganisms were closed with a sterile plate sealer and incubated for $72 \mathrm{~h}$ in an anaerobic chamber, in 5-10\% $\mathrm{H}_{2}, 10 \% \mathrm{CO}_{2}, 80-85 \% \mathrm{~N}_{2}$ atmosphere, at $36^{\circ} \mathrm{C}$. After that, resazurin $(30 \mu \mathrm{L})$ in aqueous solution $(0.01 \%)$ was added to the microplates to indicate microorganism viability for MIC determination. Chlorhexidine dihydrochloride (CHD) was used as a positive control, and the concentrations ranged from 0.115 to $59 \mu \mathrm{g} / \mathrm{mL}$. Controls were also performed to determine the sterility of the TSB and Schaedler broths, control culture (inoculum), CHD, the derivatives $\mathbf{4}$, and control DMSO. The MIC values were determined as the lowest concentration of derivatives $\mathbf{4}$ capable of inhibiting the growth of the microorganisms.

For Mycobacterium spp. the antimicrobial activity of the derivatives 4 was evaluated in vitro using the microplate microdilution technique (Palomino et al. 2002), using resazurin as an indicator of microbial activity (REMAResazurin Microtiter Assay), which allowed the determination of the minimum inhibitory concentration (MIC) against the microorganisms evaluated. The compounds were dissolved in dimethyl sulfoxide (DMSO) and serially diluted in Middlebrook 7 $\mathrm{H} 9$ broth prior to inoculation, the final concentration of DMSO being $<0.3 \%$. The inoculum was adjusted to each organism to produce a cellular concentration of $10^{8}$ colony forming units $(\mathrm{CFU} / \mathrm{mL})$. The concentrations of the compounds tested ranged from 0.195 to $200 \mu \mathrm{g} / \mathrm{mL}$ for the bacteria and from 31.5 to $2000 \mu \mathrm{g} / \mathrm{mL}$ for Mycobacterium. Microplates (96 wells) were incubated at $37^{\circ} \mathrm{C}$ for $24 \mathrm{~h}$. Thereafter, $30 \mu \mathrm{L}$ of aqueous resazurin solution $(0.01 \%)$ was added to indicate the viability of the microorganisms. The MIC was determined as the lowest concentration of the compound capable of inhibiting the growth of the microorganism. Chlorhexidine was used as the reference antibiotic at concentrations of $0.115-59.0 \mu \mathrm{g} /$ $\mathrm{mL}$ in the bioassays for bacteria and isoniazid at concentrations of $0.015-1.0 \mu \mathrm{g} / \mathrm{mL}$ for Mycobacterium. Middlebrook $7 \mathrm{H} 9$ broth containing $0.2 \%$ DMSO was used as a negative control.

Acknowledgements The authors would like to thank Fundação de Amparo à Pesquisa do Estado de São Paulo (FAPESP) (Proc. 2014/ 07493-5), for their financial support and Coordenacão de Aperfeiçoamento de Pessoal de Nível Superior (CAPES) for scholarship.

\section{Compliance with Ethical Standards}

Conflict of interest The authors declare that they have no conflict of interest.

\section{References}

Antunez-Mojica M, Rodríguez-Salarichs J, Redondo-Horcajo M, Leon A, Barasoain I, Canales A, Cañada FJ, Jiménez-Barbero J, Alvarez L, Díaz JF (2016) Structural and biochemical characterization of the interaction of tubulin with potent natural analogues of podophyllotoxin. J Nat Prod 79(8):2113-2121

Desai NC, Patel By, Dave BP (2017) Synthesis and antimicrobial activity of novel quinoline derivatives bearing pyrazoline and pyridine analogues. Med Chem Res 26(1):109-119

El-Essawy AF, El-Sayed WA (2013) Synthesis of new 1,3,4-Oxadiazol, Thiadiazole, 1,2,4-Triazole, and Arylidene Hydrazide derivatives of 4-Oxo-1,4-dihydroquinoline with antimicrobial evaluation. J Heterocycl Chem 50(S1):E1-E8

Frackenpohl J, Adelt I, Antonicek H, Arnold C, Behrmann P, Blaha N, Böhmer J, Gutbrod O, Hanke R, Hohmann S, Houtdreve M, Lösel P, Malsam O, Melchers M, Neufert V, Peschel E, Reckmann U, Schenke T, Thiesen HP, Velten R, Vogelsang K, Weiss HC (2009) Insecticidal heterolignans-Tubuline polymerization inhibitors with activity against chewing pests. Bioorg Med Chem 17:4160-4184

Grabarczyk M, Mączka W, Wińska K, Żarowska B, Aniol M (2013) Antimicrobial activity of hydroxylactone obtained by biotransformation of bromo-and iodolactone with gemdimethylcyclohexane ring. J Braz Chem Soc 24(12):1913-1919

Kharb R, Kaur H (2013) Therapeutic significance of quinoline derivatives as antimicrobial agents. Int Res J Pharm 4(3):63-69

Keri R, Patil AS (2014) Quinoline: a promising antitubercular target. Biomed Pharmacother 68(8):1161-1175

Mazur M, Bania J, Nawrot J, Klejdysz T, Wawrzeńczyk C (2016) Lactones 46. Synthesis, antifeedant and antibacterial activity of $\gamma$ lactones with a p-methoxyphenyl substituent. Pest Manag Sci 72 (3):489-496

Meléndez-Gómez CM, Kouznetsov VV (2013) Recent developments on antimicrobial quinoline chemistry. In: Méndez-Vilas A (Ed.) Microbial Pathogens And Strategies For Combating Them: Science, Technology And Education (Volume 1). Formatex Research Center, Columbia, p 666-677 
Palomino JC, Martin A, Camacho M, Guerra H, Swings J, Portaels F (2002) Resazurin microtiter assay plate simple and inexpensive method for detection of drug resistance in Mycobacterium Tuberculosis. Antimicrob Agents Chemother 46:2720-2722

Qiu H, Qian S, Head SA, Liu JO, Jin Z (2016) Insights into the structure-activity relationship of the anticancer compound ZJ101, a derivative of marine natural product superstolide A: a role played by the lactone moiety. Bioorg Med Chem Lett 6 (15):3411-3413

Shahid HA, Jahangir S, Yousuf S, Hanif M, Sherwani SK (2016) Synthesis, crystal structure, structural characterization and in vitro antimicrobial activities of 1-methyl-4-nitro-1H-imidazole. Arab J Chem 9(5):668-675

Spanò V, Parrino B, Carbone A, Montalbano A, Salvador A, Brun P, Vedaldi D, Diana P, Cirrincione G, Barraja P (2015) Pyrazolo $[3,4-\mathrm{H}]$ quinolines promising photosensitizing agents in the treatment of cancer. Eur J Med Chem 102:334-351

Teponno RB, Kusari S, Spiteller M (2016) Recent advances in research on lignans and neolignans. NatProdRep 33:1044-1092

Vandekerckhove S, D'hooghe M (2015) Quinoline-based antimalarial hybrid compounds. Bioorg Med Chem 23:5098-5119
Vandekerckhove S, Van Herreweghe S, Willems J, Danneels B, Desmet T, Kock C, Smith PJ, Chibale K, D'hooghe M (2015) Synthesis of functionalized 3-, 5-, 6- and 8-aminoquinolines via intermediate (3-pyrrolin-1-yl)- and (2-oxopyrrolidin-1-yl)quinolines and evaluation of their antiplasmodial and antifungal activity. Eur J Med Chem 92:91-102

Viegas-Junior C, Danuello A, da Silva Bolzani V, Barreiro EJ, Fraga CAM (2007) Molecular hybridization: a useful tool in the design of new drug prototypes. Curr Med Chem 14:1829-1852

Sousa RMF, Morais SAL, Vieira RBK, Napolitano DR, Guzmanb VB, Moraes TS, Cunha LCS, Martins CHG, Chang R, Aquino FJT, Nascimento EA, Oliveira A (2015) Chemical composition, cytotoxic, and antibacterial activity of the essential oil from Eugenia calycina Cambess leaves against oral bacteria. Ind Crops Prod 65:71-78

CLSI (2012a) Methods for Antimicrobial Susceptibility Testing of Anaerobic Bacteria, CLSI Document M11-A8, 8 ed. Clinical and Laboratory Standards Institute, Wayne, PA, p 39

CLSI (2012b) Methods for Dilution Antimicrobial Susceptibility Tests for Bacteria That Grow Aerobically, CLSI Document M7-A9, 9 ed. Clinical and Laboratory Standards Institute, Wayne, PA, p 63 